\title{
Magnetic coupling of planets and small bodies with a pulsar wind (Corrigendum)
}

\author{
F. Mottez ${ }^{1}$ and J. Heyvaerts ${ }^{2,1, \star}$ \\ ${ }^{1}$ LUTH, Observatoire de Paris-PSL, CNRS, Université Paris Diderot, 5 place Jules Janssen, 92190 Meudon, France \\ e-mail: fabrice.mottez@obspm.fr \\ 2 Observatoire Astronomique, Université de Strasbourg, 11 rue de l'Université, 67000 Strasbourg, France \\ A\&A, 532, A21 (2011), https://doi.org/10.1051/0004-6361/201116530 \\ A\&A, 532, A22 (2011), https://doi.org/10.1051/0004-6361/201117079 \\ A\&A, 569, A86 (2014), https://doi .org/10.1051/0004-6361/201424104
}

\begin{abstract}
Key words. plasmas - relativistic processes - methods: analytical - planets and satellites: dynamical evolution and stability pulsars: general - errata, addenda
\end{abstract}

\section{Introduction}

This paper investigates the electromagnetic interaction of a relativistic stellar wind with a planet or a smaller body in orbit around the neutron star. The interaction is based on the theory of Alfvén wings adapted to the context of relativistic winds. The 2011 paper comprises a short Section with numerical applications. This requires an estimate of the magnetic field in the pulsar wind, which depend on the magnetic flux $\Psi$ in the wind asymptotic regime. This flux is badly approximated. The present erratum corrects the consequences of this wrong estimate of $\Psi$ in three papers where it leads to specific consequences: regarding the magnetic drag acting on pulsar companions and a model of fast radio bursts (FRB). In those three papers, the physics remains unchanged, but the numerical results must be re-scaled. We also briefly discuss the theory of FRB published in 2014.

\section{Magnetic coupling of planets and small bodies with a pulsar wind}

In Mottez \& Heyvaerts (2011a), the magnetic field in the pulsar wind is expressed as a function of the magnetic flux in the asymptotic regime of the wind $\Psi=B_{0}^{r} r^{2}$, where $B_{0}^{r}$ is the local magnetic field, and $r$ is the distance to the neutron star (NS). This is an integral of motion along stream lines. At the end of Sect. 2, in view of numerical applications, it is evaluated as $\Psi=$ $B_{*}^{r} R_{*}^{2}$ where quantities with a star refer to the neutron star surface. This approximation corresponds to the monopole solution, which overestimates the wind magnetic field by orders of magnitude. Because this is an asymptotic approximation, its value must be evaluated in the domain where the field lines are wind-like, and not on the NS surface. The most admitted approximation is its value at the light cylinder Goldreich \& Julian (1969), where between the NS surface and the light cylinder (LC), the magnetic field is supposed dipolar. Then, $\Psi=B_{\mathrm{LC}}^{r} r_{\mathrm{LC}}^{2}=B_{*} R_{*}^{3} \Omega_{*} / c$ where $\Omega$ is the NS angular velocity, and $c$ is the speed of light. Then the toroidal component of the pulsar wind magnetic field is approximated by $B_{0}^{\phi}=B_{*} R_{*}^{3} \Omega_{*}^{2} / r c^{2}$ where $r \gg r_{\mathrm{LC}}$. The

\footnotetext{
$\star$ Deceased.
}

numerical estimates of the potential drop $U$ associated with PSR $1257+21$ and PSR 1620-26 in the end of Sect. 2 are not correct. Their right values as well as the corrected values of Table 3 in Mottez \& Heyvaerts (2011a) are given in four first columns of the present Table 1 .

\section{A magnetic thrust action on small bodies orbiting a pulsar}

In Mottez \& Heyvaerts (2011b), the same wrong evaluation of $\Psi$ leads to an overestimate of the magnetic thrust acting on bodies orbiting a pulsar described in the joint paper.

Table 1 in Mottez \& Heyvaerts (2011b) must be replaced by Table 1 of the present erratum. The conclusion is that even for small bodies orbiting the pulsars given in example, the influence of this effect on the orbit is totally negligible. Figures 2,3 , and 4 must be replaced by flat functions showing no significant evolution of the eccentricity and of the semi-major axis.

\section{Radio emissions from pulsar companions: a refutable explanation for galactic transients and fast radio bursts}

In Mottez \& Zarka (2014), because of the new evaluation of $\Psi$, Eq. (1) must be replaced by

$B_{0}^{\phi}=B_{0}^{r} \frac{v_{0}^{\phi}-\Omega_{*} r}{v_{0}^{r}} \sim-\frac{B_{0}^{r} \Omega_{*} r}{c}=-\frac{B_{*}^{r} \Omega_{*}^{2} R_{*}^{3}}{r c^{2}}$,

and in the same section, the current in the Alfvén wing for an Earth-like orbiting body at $0.2 \mathrm{AU}$ for a $1 \mathrm{~s}$ standard pulsar is not $10^{11}$ A but $I_{\mathrm{A}}=0.2310^{8} \mathrm{~A}$. For the same body orbiting a recycled pulsar, $I_{\mathrm{A}}=10^{9} \mathrm{~A}$ must be replaced by $I_{\mathrm{A}}=0.2310^{8} \mathrm{~A}$. In Sect. 4.3 (frequencies), the correct value of $\Psi$ is $\Psi=B_{*} R_{*}^{3} \Omega_{*} / c$, and Eq. (15) becomes

$f_{\mathrm{c}, \mathrm{s}}=\frac{q B_{*} \Omega_{*}}{2 \pi m c} \frac{R_{*}^{3}}{r^{2}} \sqrt{1+\left(\frac{r}{\gamma r_{\mathrm{LC}}}\right)^{2}}$, 
Table 1. Electric potential drop, total electric current associated to the Alfvén wing.

\begin{tabular}{llllllc}
\hline \hline Name & $U$ & $I$ & $\dot{E}_{\text {Jmax }}$ & $\Delta a /$ year & $\Delta e /$ year & $D / \sqrt{G M}$ \\
\hline Unit & $(\mathrm{V})$ & $(\mathrm{A})$ & $(\mathrm{W})$ & $\left({\left.\mathrm{m} . \mathrm{yr}^{-1}\right)}^{-}\right)$ & $\left(\mathrm{yr}^{-1}\right)$ & $\left(\mathrm{m}^{5 / 2} \cdot \mathrm{s}^{-1}\right)$ \\
\hline PSR 1257+12 a & $4.010^{10}$ & $1.110^{8}$ & $3.410^{18}$ & $1.510^{-7}$ & 0 & $2.110^{3}$ \\
PSR 1257+12 b & $1.310^{11}$ & $3.410^{8}$ & $3.410^{19}$ & $1.310^{-8}$ & $3.310^{-21}$ & $3.410^{2}$ \\
PSR 1257+12 c & $9.710^{10}$ & $2.610^{8}$ & $1.910^{19}$ & $1.010^{-8}$ & $2.810^{-21}$ & $3.510^{2}$ \\
PSR 1620-26 a & $1.910^{10}$ & $3.010^{7}$ & $2.810^{17}$ & $6.710^{-11}$ & 0 & $1.110^{2}$ \\
PSR 1s b 10,000 km & $8.410^{9}$ & $2.210^{7}$ & $1.510^{17}$ & $6.310^{-9}$ & 0 & $9.910^{1}$ \\
PSR 10ms 100 km & $1.610^{8}$ & $4.210^{5}$ & $5.110^{13}$ & $2.610^{-8}$ & $3.110^{-19}$ & $1.710^{2}$ \\
PSR 10ms 1 km & $1.610^{6}$ & $4.210^{3}$ & $5.110^{9}$ & $2.610^{-6}$ & $3.110^{-17}$ & $1.710^{4}$ \\
PSR 1s b 100 km & $1.610^{8}$ & $4.210^{5}$ & $5.110^{13}$ & $2.610^{-6}$ & $3.110^{-17}$ & $1.710^{4}$ \\
PSR 1s b 1 km & $1.610^{6}$ & $4.210^{3}$ & $5.110^{9}$ & $2.610^{-4}$ & $3.110^{-15}$ & $1.710^{6}$ \\
\hline
\end{tabular}

Note. Electrical energy $\dot{E}_{\text {Jmax }}$ dissipated in the Alfvén wing. Variation per (terrestrial) year of the semi-major axis, variation of the eccentricity, per year, $\Delta e /$ year), and the coefficient $D / \sqrt{G M}$ that scales these computations.

where $\gamma$ is the wind Lorentz factor, and $R_{\mathrm{LC}}$ is the light cylinder radius. Equation (16) becomes

$$
\begin{aligned}
f_{\mathrm{c}, \mathrm{o}}=0.52 \gamma & \left(\frac{B_{*}}{10^{5} \mathrm{~T}}\right)\left(\frac{1 \mathrm{AU}}{r}\right)^{2}\left(\frac{R_{*}}{10^{4} \mathrm{~m}}\right)^{3}\left(\frac{10 \mathrm{~ms}}{T_{*}}\right) \times \\
& \left\{1+\left[\frac{\pi 10^{5}}{\gamma}\left(\frac{10 \mathrm{~ms}}{T_{*}}\right)\left(\frac{r}{1 \mathrm{AU}}\right)\right]^{2}\right\}^{1 / 2} .
\end{aligned}
$$

In Sect. 4.4 (brightness), Eq. (18) becomes

$\dot{E}_{J}=\frac{\pi}{\mu_{0} c^{3}} R_{b}^{2} r^{-2} R_{*}^{6} B_{*}^{2} \Omega_{*}^{4}$,

where $R_{b}$ is the radius of the body orbiting the NS. Equation (19) becomes

$P_{\text {radio }}=\epsilon \dot{E}_{J}=\epsilon \frac{\pi}{\mu_{0} c^{3}} R_{b}{ }^{2} r^{-2} R_{*}^{6} B_{*}^{2} \Omega_{*}^{4}$,

and Eq. (20) becomes

$$
\begin{aligned}
\left(\frac{<S>}{\mathrm{Jy}}\right)= & 2.710^{-3}\left(\frac{\gamma}{10^{5}}\right)^{2}\left(\frac{\epsilon}{10^{-3}}\right)\left(\frac{R_{b}}{10^{7} \mathrm{~m}}\right)^{2} \times \\
& \left(\frac{1 \mathrm{AU}}{r}\right)^{2}\left(\frac{R_{*}}{10^{4} \mathrm{~m}}\right)^{6}\left(\frac{B_{*}}{10^{5} \mathrm{~T}}\right)^{2} \times
\end{aligned}
$$

$$
\left(\frac{10 \mathrm{~ms}}{T_{*}}\right)^{4}\left(\frac{\mathrm{Mpc}}{D}\right)^{2}\left(\frac{1 \mathrm{GHz}}{\Delta f}\right)
$$

In Fig. 6, the frequencies (vertical axis) must be reduced by a factor $2.110^{-2}$. In Fig. 7, they must be rescaled by a factor $2.110^{-4}$. In Fig. 8, the distances from where a $1 \mathrm{Jy}$ signal can be observed must be rescaled by a factor $2.110^{-2}$.

In the second paragraph of Sect. 5.1, it is said that a recycled millisecond pulsar and a planet corresponding to the reference values of Eq. (20) except $\gamma=10^{6}$ could provide a FRB signal seen from a distance of $1 \mathrm{Gpc}$. This is wrong. Actually, a young millisecond pulsar, characterized by the reference values of Eq. (20), except $\gamma=10^{6}$ and $B_{*}=10^{7}$ T could provide the required FRB characteristics.

In Sect. 5.2, third paragraph, it is said erroneously that a kilometer-sized asteroid orbiting a standard pulsar $(P=1 \mathrm{~s}$, $B_{*}=10^{8} \mathrm{~T}$ ) could explain the PSR J1928+15 event. Actually, the same asteroid orbiting a highly magnetized pulsar $(P=1 \mathrm{~ms}$, $B_{*}=10^{9} \mathrm{~T}$ ) could explain this phenomenon.

\section{References}

Goldreich, P., \& Julian, W. H. 1969, ApJ, 157, 869 Mottez, F., \& Heyvaerts, J. 2011a, A\&A, 532, A21 Mottez, F., \& Heyvaerts, J. 2011b, A\&A, 532, A22 Mottez, F., \& Zarka, P. 2014, A\&A, 569, A86 